\title{
Effects of Coenzyme Q10 Supplementation on Serum Lipoproteins, Plasma Fibrinogen, and Blood Pressure in Patients With Hyperlipidemia and Myocardial Infarction
}

\author{
Mona Mohseni ${ }^{1}$; Mohamad Reza Vafa ${ }^{1}$; Seyed Javad Hajimiresmail ${ }^{2}$; Mitra Zarrati ${ }^{3}$; Abbas \\ Rahimi Forushani ${ }^{4}$; Vida Bitarafan ${ }^{3}$; Farzad Shidfar ${ }^{1, *}$ \\ ${ }_{2}^{1}$ Department of Nutrition, School of Public Health, Iran University of Medical Sciences, Tehran, IR Iran \\ ${ }_{3}^{2}$ Cardiology Division, Department of Internal Medicine, School of Medicine, Iran University of Medical Sciences, Tehran, IR Iran \\ ${ }^{3}$ Department of Nutrition, School of Nutritional Sciences and Dietetics, Tehran University of Medical Sciences, Tehran, IR Iran \\ ${ }^{4}$ Department of Epidemiology and Biostatistics, School of Public Health, Tehran University of Medical Sciences, Tehran, IR Iran \\ ${ }^{*}$ Corresponding Author: Farzad Shidfar, Department of Nutrition, School of Public Health, Iran University of Medical Sciences, Tehran, IR Iran. Tel:+98-2188622721; Fax:+98-2188622721, \\ E-mail: f-shidfar@tums.ac.ir
}

Received: November 27, 2013; Revised: March 14, 2014; Accepted: April 5, 2014

\begin{abstract}
Background: Low plasma concentrations of coenzyme Q10 (CoQ10) have been associated with concentration of lipoproteins and other factors contributing to coronary heart diseases.

Objectives: The present investigation aimed to improve the blood pressure and serum lipoproteins concentration in patients with myocardial infarction (MI) by CoQ10 supplementation.

Patients and Methods: In this randomized double-blinded controlled clinical trial, 52 Iranian patients with hyperlipidemia and MI were recruited to examine the effect of CoQ10 on serum total cholesterol (TC), LDL-C, HDL-C, triglyceride (TG), LDL-C/HDL-C ratio, TC/HDL-C ratio, fibrinogen, systolic blood pressure (SBP) and diastolic blood pressure (DBP). Individuals were randomly allocated to two groups for receiving either $200 \mathrm{mg} / \mathrm{d}$ of CoQ10 or placebo for 12 weeks.

Results: There were not significant differences in serum LDL-C ( $2.70 \pm 0.31 \mathrm{vs.} 2.70 \pm 0.35 \mathrm{mmol} / \mathrm{L}), \mathrm{TC}(4.47 \pm 0.33 \mathrm{vs} .4 .93 \pm 0.57 \mathrm{mmol} / \mathrm{L})$, TG $(2.48 \pm 0.12$ vs. $2.25 \pm 0.69 \mathrm{mmol} / \mathrm{L})$, and fibrinogen $(2.08 \pm 0.99 \mathrm{vs.} 38.7 \pm 0.64 \mathrm{mg} / \mathrm{dL})$ between CoQ10 and placebo groups. After 12 weeks, a significant enhancement in serum HDL-C ( $1.44 \pm 0.18 \mathrm{vs} .1 .14 \pm 0.18 \mathrm{mmol} / \mathrm{L})$ level was observed between groups after the supplementation $(\mathrm{P}<0.001)$. A significant reduction of TC, LDL-C, and fibrinogen and a significant increase in HDL-C concentration was observed in CoQ10 group after intervention $(\mathrm{P}<0.001)$. Our assessment demonstrated statistically significant differences between the two groups in SBP and DBP after intervention $(\mathrm{P}<0.001)$. ANCOVA also revealed significant differences in the ratio of LDL-C/HDL-C and TC/HDL-C between the two groups ( $1.89 \pm 0.42$ vs. $2.39 \pm 0.38, \mathrm{P}=0.002$; and $3.2 \pm 0.5$ vs. $4.24 \pm 0.66, \mathrm{P}=0.01$, respectively). A significant reduction of LDL-C/HDL-C and TC/ HDL-C was observed in CoQ10 group $(\mathrm{P}<0.001)$.

Conclusions: Twelve-week supplementation with CoQ10 in patients with hyperlipidemia and MI can improve blood pressure, serum HDL-C as well as LDL-C/HDL-C and TC/HDL-C ratios; therefore, it might decrease the risk of frequent MI.
\end{abstract}

Keywords:Myocardial Infarction; Coenzyme Q10; Lipoprotein; Fibrinogen; Blood Pressure

\section{Background}

Asian people have the largest rate of coronary artery disease (CAD) prevalence worldwide. In addition, the correlation with lipid disorders with CAD has been proved. Hence, a large value of low-density lipoprotein cholesterol (LDL-C) is considered as a substantial indicator of CAD risk (1). Based on INTERHEART survey in Asia, there is a close link between LDL-C and myocardial infarction (MI) among Asian population. That is why this study believes that the targets and thresholds of therapy should be thoughtfully considered, even for low baseline values (1). CAD is mainly caused by modifiable risk factors; nevertheless, it remains the leading cause of morbidity and mortality globally (2). Controlling hyperlipidemia is vital in patients with cardiovascular disease (CVD) (3). Due to the lack of balance in the thrombotic- fibrinolytic equilibrium with irregular plasma fibrinogen levels, patients with atherosclerosis face with prothrombic state. In that regard, incidence of unusual changes in coagulating system among patients with unsteady CAD and considerable coronary stenosis is probable. Consequently, these patients are apt to coronary thrombotic incidence (4). Modifying lifestyle can considerably reduce fibrinogen levels, which would also affect the levels of established risk factors such as nutrients, diet, regular physical activity; therefore, measurement or modification of fibrinogen might play some roles in disease prediction or prevention (5). Notably, in randomized controlled studies, specific members of the fibrate class such as clofibrate and bezafibrate, which lower fibrinogen levels, failed to show any

Copyright ( 2014, Iranian Red Crescent Medical Journal; Published by Kowsar. This is an open-access article distributed under the terms of the Creative Commons Attribution-NonCommercial 4.0 International License (http://creativecommons.org/licenses/by-nc/4.0/) which permits copy and redistribute the material just in noncommercial usages, provided the original work is properly cited. 
beneficial effects. Coenzyme Q10 (CoQ10) is an endogenous provitamin that is also known as ubiquinone (6). It functions as a coenzyme in the energy-producing metabolic pathways; therefore, performs a fundamental function in cellular ATP synthesis process. By considering its role in respiratory chain, CoQ10 functions as an antioxidant, scavenging free radicals and inhibiting lipid peroxidation. Consequently, CoQ10 efficiency in CVD such as cardiomyopathy, hypertension (HTN), angina pectoris, and atherosclerosis has been discovered (7). The cholesterol-lowering hydroxymethylglutaryl-coenzyme A (HMG-CoA) reductase inhibitors inhibit cholesterol synthesis by repressing HMG CoA reductase and the mevalonate pathway. CoQ10 is synthesized from mevalonate and tyrosine in all cells and is one of the several end products of the mevalonate pathway. Since statin shares the hepatic mevalonate synthesis pathway with cholesterol, CoQ10 levels are reduced by statin therapy (8). Recognizing the plasma CoQ10 concentrations, which is decreased in patients taking statin, has led to the hypothesis stating that CoQ10 supplementation might be beneficial to these patients (9).

\section{Objectives}

The present study aimed to investigate the effects of CoQ10 on serum lipoproteins and blood pressure in patients with hyperlipidemia and MI after 12 weeks of CoQ10 supplementation.

\section{Patients and Methods}

This randomized double-blinded controlled clinical trial was conducted in Tehran, Iran, between August 2011 and June 2012 on Iranian individuals with hyperlipidemia (cholesterol $>5.18 \mathrm{mmol} / \mathrm{L}$, triglyceride $>1.70$ $\mathrm{mmol} / \mathrm{L}$ ) who were referred to the Hazrat Rasool hospital. Ethical approval was obtained from the Ethics Committee of Iran University of Medical Sciences in 2010. All participants had MI and were 35 to 70 years old. Exclusion criteria included smoking and alcohol consumption, diabetes mellitus, kidney and liver disorders, clinical signs of acute inflammation, infectious disease during the study time, consumption of antioxidant such as ascorbic acid, $\alpha$-tocopherol, and omega-3 supplement as well as non-steroidal anti-inflammatory drugs for three months before the study, and any changes in their drugs and their dosage during the study. Participants were instructed not to change their dietary habits throughout the study. Beta-blocker, thrombolytic, and statin drugs were administered to all patients. Written informed consent was obtained from all patients. The study protocol was approved by the Iran University of Medical Sciences. The clinical trial was registered in Iranian Registry of Clinical Trials (IRCT), number IRCT138811192709N4.

\subsection{Study Design}

This randomized, double-blinded, placebo-controlled study aimed to examine the effects of CoQ10 and placebo in patients with hyperlipidemia and MI. We considered two sided-significance levels of $5 \%$ and with $80 \%$ power, a sample size of 22 subjects per group was calculated, which was inflated to accommodate the anticipated dropout rate of $10 \%$. For predicting the missing samples in each group, we consider 27 subjects in both groups, but two patients were excluded from our study due to diabetes and liver disease (Figure 1). Therefore, the 52 enrolled patients underwent a block randomization, using a computer-generated sequence: 26 patients were allocated to the intervention group and 26 patients in the placebo group. The sample size was calculated by using the following formula:

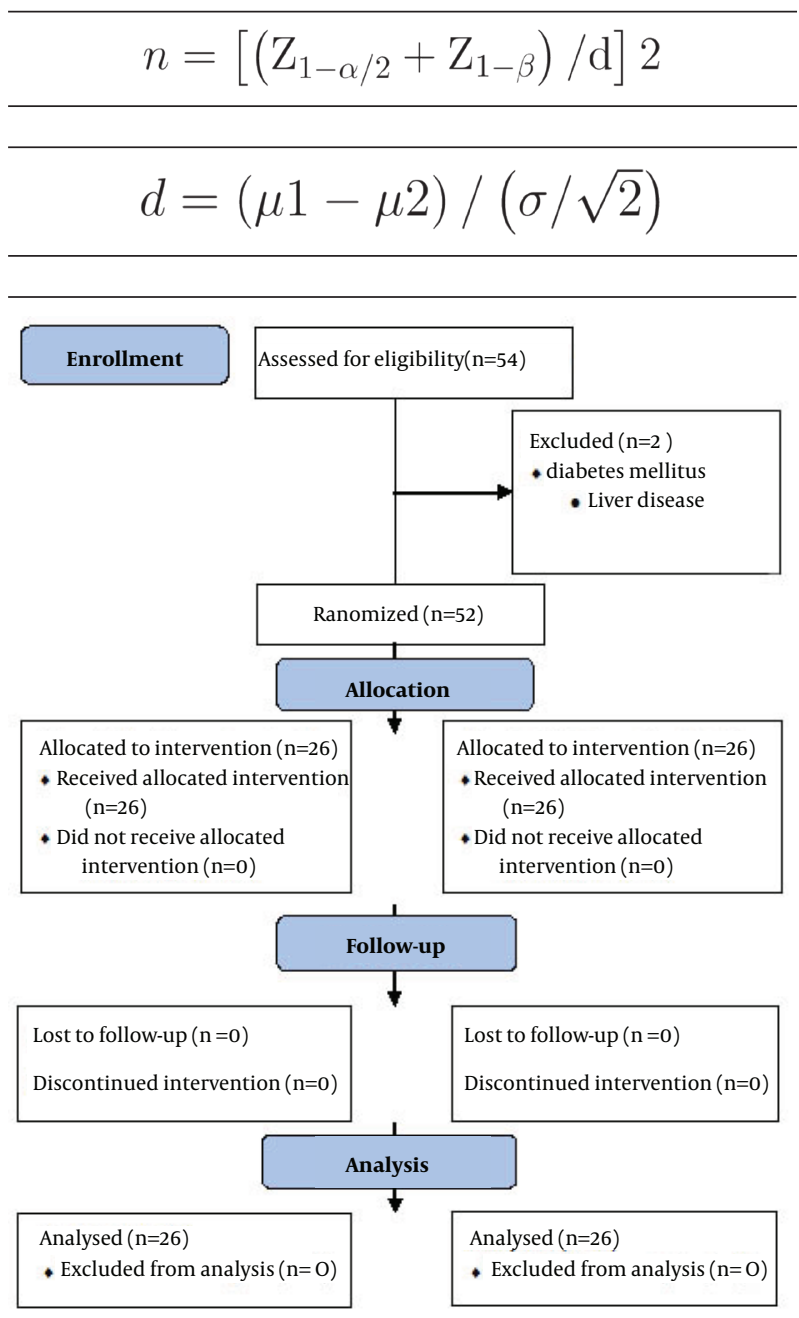

Figure 1. Flow Diagram of Patient Recruitment and Randomization Process

Subjects in CoQ10 group received two capsules of CoQ10 (Health Burst Co.) twice daily $(200 \mathrm{mg} / \mathrm{d}$ ) and placebo group received two capsules of placebo twice daily, (each capsule containing lactose powder (Health Burst Co.), for 12 weeks. Laboratory data before and after intervention were gathered. Serum total cholesterol (TC), LDL-C, high-density lipoprotein cholesterol (HDL- 
C), triglyceride (TG) levels, HDL-C/LDL-C and TC/HDL-C ratios, and plasma fibrinogen levels were determined before intervention and after 12 weeks. Physical activity was assessed by the validated International Physical Activity Questionnaire (IPAQ) (10). Participants, nutrition specialists, and external assessors were blinded to the assigned interventions to each patient. Calorie and nutrient intake were monitored by using a threeday dietary food recall at the baseline and at the end of the first, second, and third months of intervention and were analyzed by using the Nutritionist IV software (First Databank, San Bruno, CA., USA), modified for Iranian foods. Body mass index (BMI) was calculated by dividing weight $(\mathrm{Kg})$ to square of height $\left(\mathrm{m}^{2}\right)$. Systolic and diastolic blood pressure (SBP and DBP, respectively) were also measured at baseline and after the 12 weeks of intervention. Blood pressure was measured in the sitting position after 15 minutes rest.

\subsection{Laboratory Methods}

A blood sample was drawn from each participant at entry and end of the study in the morning and after at least 12 hours of fasting. Serum was also separated by centrifugation and was stored at $-70^{\circ} \mathrm{C}$. Serum levels of TC, HDL-C, LDL-C, and TG were measured enzymatically (Pars Azmoon. Co., Iran). Fibrinogen was also measured by immunoturbidimetry kit (Pars Azmoon. Co., Iran) according to the manufacturer's instructions. All measures were reported by a technician who was blind to the assigned treatment to each patient.

\subsection{Statistical Analysis}

Data were presented as mean \pm standard deviation. ANCOVA method was used to compare the effect of CoQ10 treatment on the changes of studied factors. Repeated Measures were designed to compare the food recalls taken at four periods during intervention (week 0 , week 4 , week 8 , and week 12). The significant differences between the groups at various time points were also assessed by paired-samples t test. All numeric variables were tested for normality of distribution by the Kolmogorov-Smirnov test and if necessary, were subjected to logarithmic transformation before applying parametric tests. Results were considered significant if the $P$ value was $<0.05$. The SPSS 18 (SPSS Inc., Chicago, IL, USA) was used for all statistical analyses.

\section{Results}

A total of 52 patients, 39 males (75\%) 13 females (15\%), were enrolled in the study and completed the trial. At baseline, there was no difference between subjects in CoQ10 group $(n=26)$ and those in placebo group $(n=$ 26) in age and sex distribution as well as the levels of TC, LDL-C, HDL-C, TG, and fibrinogen. The results of the 24 hours food recalls taken at four time points during the intervention are shown in Table 1. There were no significant differences between the two groups at baseline and end of the study in mean of energy, nutrients intake and physical activity.

Table 2 represents the values of anthropometric indices, SBP, and DBP values of all participants at the beginning and at the end of the intervention. There were no statistically significant differences in weight and body mass index values between the two groups after the intervention. Our results demonstrated statistically significant differences between the study groups in SBP and DBP after intervention $(\mathrm{P}<0.001)$.

Mean levels of TC, LDL-C, HDL-C, TG, and fibrinogen, had no statistically different between the two groups after the intervention (Table 3). A significant enhancement in serum HDL-C level was observed between groups after the intervention. For other variables, no statistically significant difference was observed between two groups in the end of the study. The increase in HDL-C level $(\mathrm{P}<$ $0.001)$ and decrease in all variables $(P<0.001)$, except TG $(P=0.21)$, from the baseline showed statistical significant changes in the CoQ10 group (Table 3).

As it is shown in Figure 2 a, in comparison to the baseline, the ratio of LDL-C/HDL-C decreased in both groups but this reduction was statistically significant only in CoQ10 group $(\mathrm{P}=0.01)$. ANCOVA also revealed significant differences in the ratio of LDL-C/HDL-C between the two groups $(P=0.002)$. Figure $2 \mathrm{~b}$ shows that after the 12 weeks of intervention, a significant reduction in the ratio of TC/HDL-C was observed in the CoQ10 group $(P=0.03)$. This reduction was significant between two groups after intervention $(\mathrm{P}=0.01)$.

\section{Discussion}

The antioxidant role of CoQ10, as an inhibitor of lipid peroxidation, has been known for many years. Endothelial dysfunction is an important component of MI and may depend on reduced nitric oxide (NO) synthesis, increased NO inactivation, or both. Watts et al. showed that due to the effect of CoQ10 on serum lipoproteins, supplementation with CoQ10 increases endothelial function in patients with dyslipidemia. This study provided evidence for anti-atherogenic action of CoQ10, which is exerted through improving the endothelial function of conduit arteries (11). The results of this double-blinded randomized clinical trial demonstrate that intake of CoQ10 (200 $\mathrm{mg} / \mathrm{d}$ ) for 12 weeks would lead to significant increase in serum HDL-C and significant decrease in LDL-C/HDL-C ratio and TC/HDL-C ratio in patients with MI. Our results also indicated a slight but not significant decrease in serum LDL-C, TC, and TG. TC/HDL-C ratio is a significant predictor of cardiovascular events and a therapeutic target in high-risk patients such as those with MI. Reduction in this ratio was reached by using more aggressive LDLC lowering therapy or potentially increasing the HDL-C level $(12,13)$. This increase in HDL-C was observed in our 
Mohseni M et al.

\begin{tabular}{|c|c|c|c|c|c|}
\hline Week & Coenzyme Q10 $(n=26)$ & P Value $^{\mathrm{b}}$ & Placebo $(n=26)$ & PValue $^{\mathrm{b}}$ & PValue $^{\mathrm{C}}$ \\
\hline \multicolumn{6}{|l|}{ Energy, kcal/d } \\
\hline 0 & $1555 \pm 295$ & 0.321 & $1590 \pm 343$ & $<0.001$ & 0.07 \\
\hline 4 & $1535 \pm 250$ & & $1585 \pm 343$ & & 0.08 \\
\hline 8 & $1536 \pm 527$ & & $1565 \pm 310$ & & 0.1 \\
\hline 12 & $1584 \pm 253$ & & $1573 \pm 303$ & & 0.09 \\
\hline Carbohydrate, g/d & & 0.123 & & $<0.001$ & \\
\hline 0 & $250 \pm 57$ & & $256 \pm 32$ & & 0.25 \\
\hline 4 & $253 \pm 52$ & & $251 \pm 35$ & & 0.05 \\
\hline 8 & $240 \pm 28$ & & $249 \pm 31$ & & 0.81 \\
\hline 12 & $220 \pm 30$ & & $222 \pm 31$ & & 0.06 \\
\hline Protein, $\mathrm{g} / \mathrm{d}$ & & 0.453 & & 0.45 & \\
\hline 0 & $61 \pm 14$ & & $65 \pm 12$ & & 0.66 \\
\hline 4 & $60.6 \pm 13$ & & $65.2 \pm 12$ & & 0.47 \\
\hline 8 & $61.2 \pm 12$ & & $61 \pm 10$ & & 0.58 \\
\hline 12 & $63 \pm 12$ & & $62 \pm 11$ & & \\
\hline Fat, $g / d$ & & 0.711 & & $<0.001$ & \\
\hline 0 & $68 \pm 15$ & & $61 \pm 16$ & & 0.43 \\
\hline 4 & $64 \pm 10$ & & $66 \pm 9$ & & 0.09 \\
\hline 8 & $66 \pm 8$ & & $62 \pm 9$ & & 0.12 \\
\hline 12 & $63 \pm 8$ & & $64 \pm 6$ & & 0.21 \\
\hline Cholesterol, mg/d & & 0.99 & & 0.31 & \\
\hline 0 & $137 \pm 98$ & & $139 \pm 88$ & & 0.04 \\
\hline 4 & $127 \pm 98$ & & $156 \pm 82$ & & 0.04 \\
\hline 8 & $147 \pm 68$ & & $137 \pm 112$ & & 0.37 \\
\hline 12 & $156 \pm 98$ & & $139 \pm 98$ & & \\
\hline Saturated Fat, g/d & & 0.05 & & 0.06 & \\
\hline 0 & $8.9 \pm 5.3$ & & $9 \pm 4.4$ & & 0.64 \\
\hline 4 & $9 \pm 5$ & & $9 \pm 4.4$ & & 0.02 \\
\hline 8 & $8.5 \pm 5$ & & $9.2 \pm 4$ & & 0.07 \\
\hline 12 & $8.9 \pm 4.7$ & & $8.7 \pm 5.6$ & & 0.1 \\
\hline Monounsaturated Fat, g/d & & 0.121 & & 0.423 & \\
\hline 0 & $8.9 \pm 4$ & & $8.3 \pm 7$ & & 0.15 \\
\hline 4 & $9 \pm 5$ & & $8 \pm 4$ & & 0.01 \\
\hline 8 & $9.2 \pm 4$ & & $8.9 \pm 2.8$ & & 0.002 \\
\hline 12 & $8.8 \pm 4.2$ & & $9.4 \pm 4.6$ & & 0.08 \\
\hline Polyunsaturated Fat, g/d & & 0.112 & & 0.29 & \\
\hline 0 & $16 \pm 8$ & & $16.7 \pm 8$ & & 0.48 \\
\hline 4 & $16.7 \pm 5$ & & $16.9 \pm 6$ & & 0.49 \\
\hline 8 & $15.9 \pm 4$ & & $15.8 \pm 5$ & & 0.22 \\
\hline 12 & $15.8 \pm 6.4$ & & $16.3 \pm 4.6$ & & \\
\hline Vitamin A, IU/d & & 0.313 & & 0.421 & \\
\hline 0 & $499 \pm 262$ & & $507 \pm 168$ & & 0.55 \\
\hline 4 & $495 \pm 268$ & & $512 \pm 245$ & & 0.12 \\
\hline 8 & $497 \pm 258$ & & $402 \pm 249$ & & 0.08 \\
\hline 12 & $494 \pm 268$ & & $499 \pm 300$ & & \\
\hline Vitamin C, mg/d & & 0.246 & & 0.26 & \\
\hline 0 & $68 \pm 5.3$ & & $69 \pm 3.3$ & & 0.08 \\
\hline 4 & $63 \pm 6.3$ & & $65 \pm 6.3$ & & 0.5 \\
\hline 8 & $63 \pm 5.6$ & & $66 \pm 4.3$ & & 0.1 \\
\hline 12 & $66 \pm 5.3$ & & $68 \pm 6.6$ & & 0.27 \\
\hline Vitamin E, mg/d & & 0.311 & & 0.212 & \\
\hline 0 & $12 \pm 3.4$ & & $12.5 \pm 2.1$ & & 0.07 \\
\hline 4 & $11 \pm 2.7$ & & $13 \pm 1.4$ & & 0.05 \\
\hline 8 & $12 \pm 2.1$ & & $12 \pm 2.5$ & & 0.21 \\
\hline 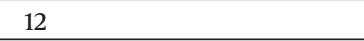 & $13 \pm 3.3$ & & $13 \pm 1.1$ & & 0.12 \\
\hline
\end{tabular}


Mohseni Met al.

\begin{tabular}{|c|c|c|c|}
\hline Week & Coenzyme Q10 $(n=26)$ & $\operatorname{Placebo}(\mathbf{n}=26)$ & PValue $^{\mathrm{C}}$ \\
\hline \multicolumn{4}{|l|}{ Age, $y$} \\
\hline 0 & $60 \pm 8$ & $61 \pm 7$ & 0.341 \\
\hline \multicolumn{4}{|c|}{ Height, cm } \\
\hline 0 & $168.3 \pm 1.3$ & $166.6 \pm 1.4$ & 0.113 \\
\hline \multicolumn{4}{|c|}{ Body Weight, kg } \\
\hline 0 & $74.69 \pm 8.91$ & $74.92 \pm 8.94$ & 0.536 \\
\hline 12 & $73.71 \pm 8.27$ & $74.03 \pm 8.87$ & \\
\hline \multicolumn{4}{|c|}{ BMI, $\mathrm{kg} / \mathrm{m}^{2}$} \\
\hline 0 & $25.91 \pm 2.53$ & $26 \pm 3.34$ & 0.222 \\
\hline 12 & $25.65 \pm 2.42$ & $26.1 \pm 3.25$ & \\
\hline \multicolumn{4}{|c|}{ SBP, mmHg } \\
\hline 0 & $143.23 \pm 2.9$ & $134 \pm 2.9$ & $\mathrm{P}<0.001$ \\
\hline 12 & $131 \pm 2.2$ & $131 \pm 1.74$ & \\
\hline \multicolumn{4}{|c|}{ DBP, $\mathrm{mmHg}$} \\
\hline 0 & $90.13 \pm 2.11$ & $100.23 \pm 1.4$ & $\mathrm{P}<0.001$ \\
\hline 12 & $82.16 \pm 2.12$ & $83 \pm 1.87$ & \\
\hline
\end{tabular}

a Abbreviations: BMI, body mass index; SBP, systolic blood pressure; and DBP, diastolic blood pressure.

$\mathrm{b}$ Values are presented as mean $\pm 2 \mathrm{SD}$.

${ }^{c}$ Pvalue for the comparison of changes after intervention between study groups (ANCOVA).

Table 3. Effect of Coenzyme Q10 on Serum Lipoproteins and Plasma Fibrinogen Concentration in the Study Groups a,b,c

\begin{tabular}{|c|c|c|c|c|c|c|c|}
\hline & \multicolumn{2}{|c|}{ Coenzyme Q10 } & \multirow[t]{2}{*}{ PValue $^{\mathrm{d}}$} & \multicolumn{2}{|c|}{ Placebo } & \multirow[t]{2}{*}{ P Value $^{\mathrm{d}}$} & \multirow[t]{2}{*}{ PValue $^{\mathrm{e}}$} \\
\hline & Week 0 & Week 12 & & Week 0 & Week 12 & & \\
\hline $\mathrm{TC}, \mathrm{mmol} / \mathrm{L}$ & $5.69 \pm 0.57$ & $4.47 \pm 0.33$ & $<0.001$ & $5.67 \pm 0.59$ & $4.93 \pm 0.57$ & $<0.001$ & 0.231 \\
\hline LDL-C, mmol/L & $3.46 \pm 0.34$ & $2.70 \pm 0.31$ & $<0.001$ & $3.47 \pm 0.34$ & $2.70 \pm 0.35$ & $<0.001$ & 0.56 \\
\hline HDL-C, mmol/L & $1.15 \pm 0.17$ & $1.44 \pm 0.18$ & $<0.001$ & $1.15 \pm 0.18$ & $1.14 \pm 0.18$ & 0.812 & $<0.001$ \\
\hline TG, mmol/L & $2.39 \pm 1.05$ & $2.48 \pm 0.12$ & 0.324 & $2.23 \pm 1.07$ & $2.25 \pm 0.69$ & 0.341 & 0.145 \\
\hline Fibrinogen, $\mathrm{g} / \mathrm{L}$ & $3.88 \pm 1.05$ & $2.08 \pm 0.99$ & 0.001 & $3.99 \pm 0.88$ & $3.87 \pm 0.64$ & 0.451 & 0.301 \\
\hline
\end{tabular}

\footnotetext{
a Abbreviations: TC, total cholesterol; LDL-C, low-density lipoprotein cholesterol; HDL-C, high-density lipoprotein cholesterol; and TG, triglyceride.

$\mathrm{b}$ Values are means $\pm 2 \mathrm{SD}$.

${ }^{\mathrm{C}} \mathrm{P}$ value $<0.05$ was considered as statistically significant.

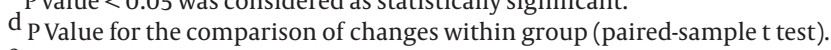

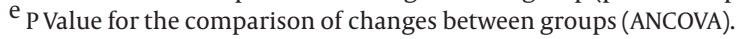

study. Patients receiving statin, show lower levels of plasma CoQ10. Therefore, statin treatment might cause CoQ10 deficiency (14). Studies have consistently demonstrated that statin therapy decreases circulating CoQ10 concentrations (15). In our study, using CoQ10 was associated with a significant increase in serum HDL-C in CoQ10 group. Except for serum HDL-C level, we did not find any significant changes in serum levels of other lipoproteins between two groups following the CoQ10 supplementation. Moreover, a significant increase was observed in the serum HDL-C levels in the CoQ10 group when compared to the placebo group. The rise in the serum HDL-C initiates cholesterol efflux and facilitates the removal of excess cholesterol from the arteries and delivers it to liver for being removed through reverse cholesterol transport pathway (16). The significant decreases in LDL-C/HDL-C ratio and TC/HDL-C ratio in our study were a favorable result for decreasing the CVD risk. However, the effects of CoQ10 on serum lipoproteins have been examined, but its effects on apolipoproteins have not been reported. According to Chapidze et al. (17), treatment with CoQ10 in patients with ischemic heart disease is associated with its potential independent role in lowering the markers of oxidative stress and decreasing the TC/HDL-C ratio. Plasma fibrinogen was not reduced by CoQ10 supplement therapy. Our results were in agreement with those of Kato et al. (18), who showed that showed the CoQ10 supplementation in patient with ischemic heart disease did not influence serum fibrinogen concentration. In addition, serum fibrinogen 
A

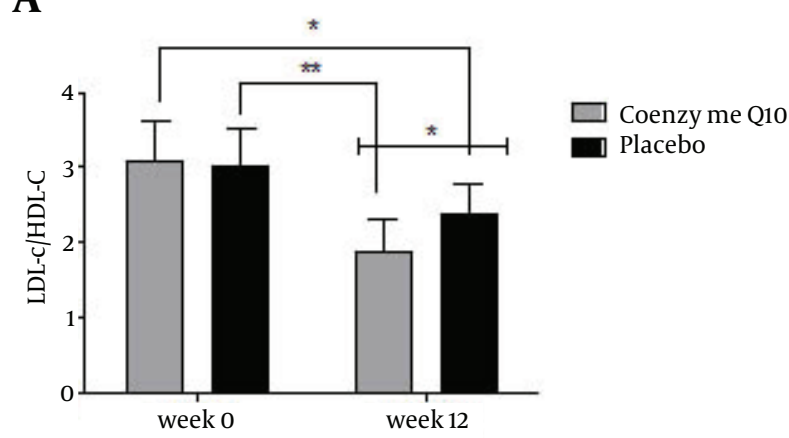

B

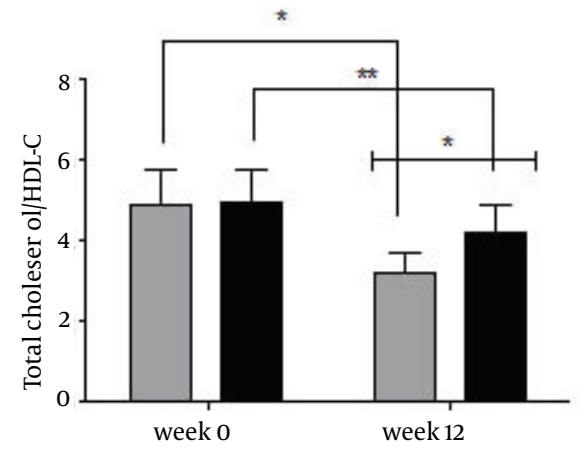

Figure 2. Changes in Low-Density to High-Density Lipoprotein Cholesterols Ratio (2a) and Total to High-Density Lipoprotein Cholesterol Ratio (2b) After Twelve Weeks of Intervention in Studies Groups $\left({ }^{*} \mathrm{P}<0.05\right.$, ${ }^{* *} \mathrm{P}$ $\geq 0.05$ )

levels did not change in patients with type 2 diabetes who were treated by $200 \mathrm{mg} /$ day CoQ10 (19). Atherosclerosis and vascular events are influenced by fibrinogen. It can induce proliferation of vascular smooth muscles through binding to LDL. On the other hand, it stimulates platelet aggregation in vessels by launching occlusive arterial blood clot (20). As elevated concentration of fibrinogen increases blood viscosity and slows blood flow, it causes thrombus formation considerably (21). The great amounts of HDL-C raises fibrinolysis and inhibits platelet aggregation and LDL-C oxidation (22). As the duration of our intervention was limited, fibrinogen concentration was not different between groups. Hence, CoQ10 takes a long time to reduce lipid in patients with MI. Based on our results, CoQ10supplement therapy in MI reduced SBP and DBP, which was in agreement with other reports (23). In several studies, the effect of CoQ10 on lowering blood pressure was shown (24-26); therefore, there is a high correlation between CoQ10 deficiency and HTN. In other word, following oral administration of CoQ10 to patients with HTN, the blood pressure will fall (27). In patients with CAD, HTN and endothelial dysfunction are alleviated due to CoQ10 supplementation. Thus, endothelial dysfunction in patients with MI can be improved by CoQ10 supplement therapy, which can modify local vascular oxidative stress (28). Furthermore, the extracellular superoxide dismutase (ecSOD) activity and endothelium-dependent vasodilation are remarkably improved by CoQ10 supplementation in those with low initial endotheliumbound ecSOD, who were already more predisposed to oxidative stress (29). Consequently, it has been suggested that CoQ10 might also indirectly affect vascular function via inhibiting the inactivation of endothelial NO, which responds rapidly to superoxide radicals. It might be due to the rate of NO inactivation by superoxide anions, that might reduce CoQ10 under high-oxidative stress condition such as chronic heart failure and facing with multiple CAD risk factors (30). Antioxidant properties of CoQ10 result in reducing free radicals, which cause inactivation of endothelium-derived relaxing factor and/or fibrosis of arteriolar smooth muscle (31). The main strengths of this study were study size, homogeneity of the study population, and strict exclusion criteria, which reduced the potential effects of confounding factors. This study was the first research about the effect of CoQ10 supplementation in patients with MI who were under statins therapy. Nonetheless, our study had some limitations and therefore, our findings should be interpreted carefully. Markers such as fibrinogen, vitamin $\mathrm{D}$, and cystatin $\mathrm{C}$ have a strong association with CVD but have not been implemented in clinic yet (32). No improvement in external validity in our study was another limitation to our trial.

In Conclusion, our study showed that supplementation with $200 \mathrm{mg} / \mathrm{d}$ CoQ10 might reduce serum HDL-C, LDL-C/ HDL-C ratio, TC/HDL-C ratio, SBP, and DBP in patients with hyperlipidemia and MI.

\section{Acknowledgements}

We would like to express our sincere thanks to Iran University of Medical Sciences for providing fund for the project as well as all patients and their medical teams.

\section{Authors' Contributions}

Dr Farzad Shidfar, Dr. Mohamad Reza Vafa, Mona Mohseni contributed to the study design, coordination of the project, and interpretation of data. Mona Mohseni and Dr. Mitra Zarrati collected data and performed data analysis. Dr. Abbas Rahimi Forushani performed sample size estimation and statistical analysis. Dr. Seyed Javad Hajimiresmail actively participated in project design. Vida Bitarafan wrote the manuscript and Dr. Farzad Shidfar performed the final revise of manuscript.

\section{Funding/Support}

This study was supported in part by grant from the Iran University of Medical Sciences.

\section{References}

1. Karthikeyan G, Teo KK, Islam S, McQueen MJ, Pais P, Wang X, et al. Lipid profile, plasma apolipoproteins, and risk of a first myocar- 
Mohseni M et al.

dial infarction among Asians: an analysis from the INTERHEART Study. J Am Coll Cardiol. 2009;53(3):244-53.

2. Shidfar F, Keshavarz A, Jallali M, Miri R, Eshraghian M. Comparison of the effects of simultaneous administration of vitamin C and omega-3 fatty acids on lipoproteins, apo A-I, apo B, and malondialdehyde in hyperlipidemic patients. Int J Vitam Nutr Res. 2003;73(3):163-70

3. Nordestgaard BG, Chapman MJ, Ray K, Boren J, Andreotti F, Watts GF, et al. Lipoprotein(a) as a cardiovascular risk factor: current status. Eur Heart J. 2010;31(23):2844-53.

4. Figueras J, Monasterio J, Domingo E, Meneses B, Nieto E, Cortadellas J, et al. Prothrombotic profile in patients with vasospastic or non vasospastic angina and non significant coronary stenosis. Thromb J. 2011;9:10.

5. Shojaie M, Pourahmad M, Eshraghian A, Izadi HR, Naghshvar F. Fibrinogen as a risk factor for premature myocardial infarction in Iranian patients: a case control study. Vasc Health Risk Manag. 2009;5:673-6.

6. Levy HB, Kohlhaas HK. Considerations for supplementing with coenzyme Q10 during statin therapy. Ann Pharmacother. 2006;40(2):290-4.

7. Ankola DD, Viswanad B, Bhardwaj V, Ramarao P, Kumar MN. Development of potent oral nanoparticulate formulation of coenzyme Q10 for treatment of hypertension: can the simple nutritional supplements be used as first line therapeutic agents for prophylaxis/therapy? EurJ Pharm Biopharm. 2007;67(2):361-9.

8. Nahas R. Complementary and alternative medicine approaches to blood pressure reduction: An evidence-based review. Can Fam Physician. 2008;54(11):1529-33.

9. Mabuchi H, Nohara A, Kobayashi J, Kawashiri MA, Katsuda S, Inazu A, et al. Effects of CoQ10 supplementation on plasma lipoprotein lipid, CoQ10 and liver and muscle enzyme levels in hypercholesterolemic patients treated with atorvastatin: a randomized double-blind study. Atherosclerosis. 2007;195(2):e182-9.

10. Hagstromer M, Oja P, Sjostrom M. The International Physical Activity Questionnaire (IPAQ): a study of concurrent and construct validity. Public Health Nutr. 2006;9(6):755-62.

11. Watts GF, Playford DA, Croft KD, Ward NC, Mori TA, Burke V. Coenzyme Q(10) improves endothelial dysfunction of the brachial artery in Type II diabetes mellitus. Diabetologia. 2002;45(3):420-6.

12. Barter P, Gotto AM, LaRosa JC, Maroni J, Szarek M, Grundy SM, et al. HDL cholesterol, very low levels of LDL cholesterol, and cardiovascular events. $N$ Engl J Med. 2007;357(13):1301-10.

13. Wang TD, Chen WJ, Chien KL, Seh-Yi Su SS, Hsu HC, Chen MF, et al. Efficacy of cholesterol levels and ratios in predicting future coronary heart disease in a Chinese population. Am J Cardiol. 2001;88(7):737-43.

14. Langsjoen PH, Langsjoen AM. The clinical use of HMG CoA-reductase inhibitors and the associated depletion of coenzyme Q10. A review of animal and human publications. Biofactors. 2003;18(14):101-11.

15. Marcoff L, Thompson PD. The role of coenzyme Q10 in statinassociated myopathy: a systematic review. J Am Coll Cardiol. 2007;49(23):2231-7

16. Kashyap M. Mechanistic studies of high-density lipoproteins.
Ame J cardiol. 1998;82(12):42U-8U.

17. Chapidze G, Kapanadze S, Dolidze N, Bachutashvili Z, Latsabidze N. Prevention of coronary atherosclerosis by the use of combination therapy with antioxidant coenzyme Q10 and statins. Georgian Med News. 2005(118):20-5

18. Kato T, Yoneda S, Kako T, Koketsu M, Hayano I, Fujinami T. Reduction in blood viscosity by treatment with coenzyme Q10 in patients with ischemic heart disease. Int J Clin Pharmacol Ther Toxicol. 1990;28(3):123-6.

19. Playford DA, Watts GF, Croft KD, Burke V. Combined effect of coenzyme Q10 and fenofibrate on forearm microcirculatory function in type 2 diabetes. Atherosclerosis. 2003;168(1):169-79.

20. Heinrich J, Assmann G. Fibrinogen and cardiovascular risk. J Cardiovasc Risk. 1995;2(3):197-205.

21. Kaur S, Singh P, Indu V, Singla G. Fibrinogen, Lp(a), Microalbuminuria and Left Ventricular Mass Index: Cardiovascular Disease Risk factors in Diabetes. Indian J Clin Biochem. 2012;27(1):94-6.

22. Jin FY, Kamanna VS, Kashyap ML. Niacin decreases removal of high-density lipoprotein apolipoprotein A-I but not cholesterol ester by Hep G2 cells. Implication for reverse cholesterol transport. Arterioscler Thromb Vasc Biol. 1997;17(10):2020-8.

23. Singh RB, Niaz MA. Serum concentration of lipoprotein(a) decreases on treatment with hydrosoluble coenzyme Q10 in patients with coronary artery disease: discovery of a new role. Int JCardiol.1999;68(1):23-9.

24. Langsjoen P, Langsjoen P, Willis R, Folkers K. Treatment of essential hypertension with coenzyme Q10. Mol Aspects Med. 1994;15 Suppl:S265-72.

25. Singh RB, Niaz MA, Rastogi SS, Shukla PK, Thakur AS. Effect of hydrosoluble coenzyme Q10 on blood pressures and insulin resistance in hypertensive patients with coronary artery disease. $J$ Hum Hypertens. 1999;13(3):203-8.

26. Burke BE, Neuenschwander R, Olson RD. Randomized, doubleblind, placebo-controlled trial of coenzyme Q10 in isolated systolic hypertension. South Med J. 2001;94(11):1112-7.

27. Houston MC. Treatment of hypertension with nutraceuticals, vitamins, antioxidants and minerals. Expert Rev Cardiovasc Ther. 2007;5(4):681-91.

28. Hamilton SJ, Chew GT, Watts GF. Coenzyme Q10 improves endothelial dysfunction in statin-treated type 2 diabetic patients. Diabetes Care. 2009;32(5):810-2.

29. Tiano L, Belardinelli R, Carnevali P, Principi F, Seddaiu G, Littarru GP. Effect of coenzyme Q10 administration on endothelial function and extracellular superoxide dismutase in patients with ischaemic heart disease: a double-blind, randomized controlled study. Eur Heart J. 2007;28(18):2249-55.

30. Belardinelli R, Tiano L, Littarru GP. Oxidative stress, endothelial function and coenzyme Q10. Biofactors. 2008;32(1-4):129-33.

31. Ignarro LJ. Biological actions and properties of endotheliumderived nitric oxide formed and released from artery and vein. Circ Res. 1989;65(1):1-21.

32. van Holten TC, Waanders LF, de Groot PG, Vissers J, Hoefer IE, Pasterkamp G, et al. Circulating biomarkers for predicting cardiovascular disease risk; a systematic review and comprehensive overview of meta-analyses. PLoS One. 2013;8(4). 